\title{
Rule-Based Ventral Cavity Multi-Organ Automatic Segmentation in CT Scans
}

\author{
Assaf B. Spanier \\ School of Eng. and Computer Science \\ The Hebrew Univ. of Jerusalem, Israel \\ assaf.spanier@mail.huji.ac.il
}

\author{
Leo Joskowicz \\ School of Eng. and Computer Science \\ The Hebrew Univ. of Jerusalem, Israel \\ josko@cs.huji.ac.il
}

\begin{abstract}
We describe a new method for the automatic segmentation of multiple organs of the ventral cavity in CT scans. The method is based on a set of rules that determine the order in which the organs are isolated and segmented, from the simplest one to the most difficult one. First, the body is isolated from the background. Second, the trachea and the left and right lungs are segmented based on their air content. Third, the spleen and the kidneys - the organs with high blood content - are segmented. Finally, the kidney is segmented based on the surrounding organs segmentation. Each organ is individually segmented with a four-step procedure that consists of: 1) definition of an inclusive region of interest; 2) identification of the largest axial cross-section slice; 3 ) removal of background structures by morphological operations, and; 4) $3 \mathrm{D}$ region growing segmentation. Our method is unique in that it uses the same generic segmentation approach for all organs and in that it relies on the segmentation difficulty of organs to guide the segmentation process. Experimental results on 15 CT scans of the VISCERAL Anatomy2 Challenge training datasets yield a Dice volume overlap similarity score of 79.1 for the trachea, 97.4 and 97.6 for the left and right lungs, 89.2 for the spleen, and 92.8 for the left kidney. For the 5 CT scans test datasets, the Dice scores are 97.9, 97.0, 85.6, 93.4 and 90.2, respectively. Our method achieved an overall DICE score of 92.8 and was ranked first among the five methods that participated in the challenge.
\end{abstract}

Copyright (C) by the paper's authors. Copying permitted only for private and academic purposes.

In: O. Goksel (ed.): Proceedings of the VISCERAL Organ Segmentation and Landmark Detection Benchmark at the 2014 IEEE International Symposium on Biomedical Imaging (ISBI), Beijing, China, May $1^{\text {st }}, 2014$ published at http://ceur-ws.org 


\section{Introduction}

The increasing amount of medical imaging data acquired in clinical practice constitutes a vast database of untapped diagnostically relevant information. Today, only a small fraction of this information is used during clinical routine or research due to the complexity, richness, high dimensionality, and data size [1].

Content-based image retrieval (CBIR) techniques have been proposed to access this information and to identify similar cases to assist radiologists in the clinical decision support process [2]. The segmentation of individual ventral cavity organs in CT scans is expected to improve the diagnostic accuracy and performance of CBIR systems. While the manual delineation of these organs is considered the gold standard, this is a tedious and very time-consuming process which is impractical for all but a few dozen datasets for research. Consequently, a plethora of methods for automatic segmentation of ventral body cavity organs in CT scans have been proposed. Liver segmentation methods are thoroughly summarized and reviewed by Mharib et al. [4]. Lungs segmentation from CT scans has been addressed by Sluimer et al. [5]. Kidney segmentation methods are described in Freiman et al [6]. While very different from each other, all these methods target a single organ and do not use information about other organs' segmentations. Thus, multi-organ segmentation requires a specific method for each organ, which yields variable quality results and quickly becomes unmanagable as the number of organs to be segmented grows. It is thus desirable to develop a single, generic approach that can be customized for each organ and that uses the information about other organs' segmentations.

The rule-based approach to medical image segmentation calls for using each organ anatomical context and prior knowledge about its location and its extension for enhancing, improving, and automating the segmentation process. In this pipeline-oriented approach, the organs of interest are successively extracted from the CT scan. Previous research has focused mainly on liver segmentation [3]. In this paper, we extend and generalize the rule-based approach to the automatic segmentation of multiple ventral cavity organs in CT scans.

\section{Method}

The basic premise of the rule-based paradigm is to sequentially extract different organs based on prior information on the organs of interest and their characteristics in the CT scan. Simple and context-free organs are segmented first, followed by more complex and context-based identfication and delineation. Our proposed approach extends the established rule-based approach by providing a unified, generic four-step approach that is customized for each organ and incorporates information about other organs prior segmentations.

\subsection{Generic organ segmentation framework}

In our generic framework, the segmentation of each organ is performed in four successive steps (Fig. 1):

1. Definition of the organ's Binary Inclusive Region Of Interest (BI-ROI) based on the target organ intensity values.

2. Identification of the organ's Largest Axial Cross Section Slice (LACSS). This is the CT scan slice where the organ has the largest axial area.

3. Removal of remaining background structures from the LACSS by morphological operations.

4. Organ segmentation by 3D region growing starting from the LACSS inside the BI-ROI. 


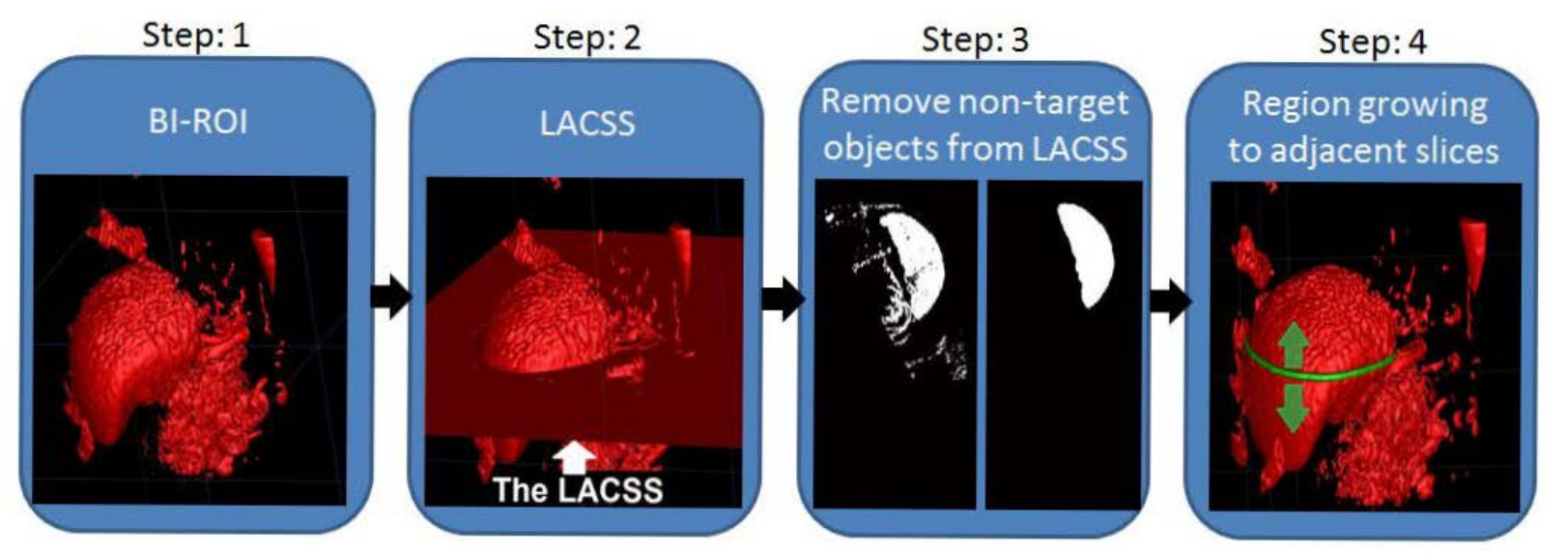

Figure 1: The four steps of the generic organ segmentation framework exemplified on the spleen.

We start with a preprocessing step that isolates the patient body from the background (air and scan gantry) based on location and intensity values. The generic four-step framework is then applied to the ventral body cavity organs in the following order. First, the breathing system organs are segmented: the trachea and the left and right lungs. Next, the organs with high blood content are segmented: the spleen, the liver, and the left and right kidneys. This organ segmentation order prevents ambiguous assignment of the same image region to multiple organs, as previously segmented image regions are excluded from the segmentation process. Due to space limitations, we illustrate below each step for the breathing system only.

\section{$2.2 \quad$ Breathing system segmentation}

The breathing system consists of the trachea and the left and right lung.

\section{Step 1: Definition of the BI-ROI: Binary Inclusive Region of Interest}

We perform a simple thresholding with the Houndsfiel Unit (HU) of air and fat $(<-500 \mathrm{HU})$. This results in a binary map consisting of air, fat, and other background structures. Then, the trachea and the lungs are separated from the from the undesired surrounding fat by finding the the largest connected component. The resulting structure includes the breathing system defines the trachea and lungs BI-ROI (Fig. 2a). This BI-ROI is further refined for the trachea and the left and right lungs.

\section{Step 2: Identification of the LACSS: Largest Axial Cross Section Slice}

The Largest Axial Cross Section Slice (LACSS) of the trachea and the lungs are identified by finding the $\mathrm{CT}$ slices in the BI-ROI with the narrowest and widest perimeters, respectively (Figs. $2 \mathrm{~b}$ and 2c). Note that the lungs slice contains two connected components, for the left and right lungs.

\section{Step 3: Removal of background structures}

No further background removal is required for the trachea and lungs, since the lungs LACSS contains exactly two connected components corresponding to the left and right lungs and the trachea LACSS contains exactly one connected component (Fig. 2). 




(a)

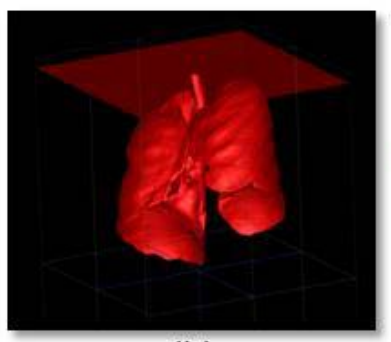

(b)

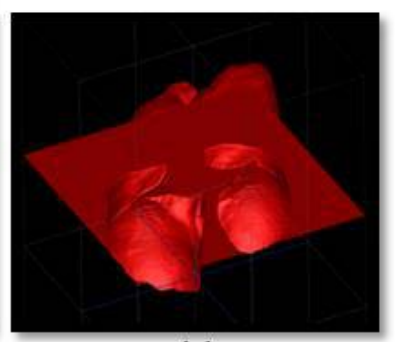

(c)

Figure 2: Illustration of the results of the first two steps of the generic organ segmentation framework on the breathing system: a) Binary Inclusive Region of Interest; b) Largest Axial Cross Section Slice plane for the trachea, and c) Largest Axial Cross Section Slice plane for the lungs.

\section{Step 4: Segmentation by 3D region growing}

The trachea and the left and right lungs are segmented by 3D region growing. The process starts at the LACSS and proceeds to adjacent CT slices withing the volume defined by the BI-ROI.

First, the distance map between the LACSS contour (Fig 3a) and the adjacent slice (Fig 3b) is computed, with the LACSS contour distance set to 0 (Fig 3c). Next, the adjacent slice and the distance map are intersected to identify regions of high change (Fig 3d). In the resulting intersection, we define a series of windows along the contour (Fig 3e) and compute the intensity histogram in each window. Finally, windows whose histograms have a positive kurtosis are considered as segmentation leakage, e.g. windows $R_{1}$ and $R_{2}$ in Fig 3e. These windows contain undesired structures whose voxels are removed from the organ segmentation. Windows whose histograms have zero or negative kurtosis, e.g. window $R_{3}$ in Fig 3e, are considered smooth and are thus segmented as part of the organ of interest. This process is repeated throughout the slices of the image until the number of segmented pixels in the slice is below a predefined threshold.

The idea behind this step is that ventral cavity organs are relatively smooth, so two adjacent slices of the same organ cannot exceed some level of variability. From a geometric point of view, we constrain the expansion of the region growing boundary curve to have variable speed according to the context. Our method takes into account the local geometry of the curvature: when the magnitude of the curvature is above a predefined threshold, we stop its propagation and allow it to continue only in low curvature regions. The rationale is once again that ventral body cavity organs should preserve some level of smoothness constraint. The final result is the $3 \mathrm{D}$ segmentation of the organ (Fig 4).

\section{Experimental Results}

We evaluated our method on two sets of scans of the VISCERAL Anatomy2 Challenge. The training and test datasets consist of 15 and 5 CT clinical scans, respectively, acquired in 2004-08. Datasets of patients younger than 18 years were not included following the recommendation of the local ethical committee (S-465/2012, approval date Feb. 21th 2013). The CT scans in-plane resolution is 0.604-0.793/0.604-0.793mm; the in-between plane resolution is $>=3 \mathrm{~mm}$. A VISCERAL team radiologist manually produced ground-truth segmentations for each scan.

Table 1 summarizes the results for each type of dataset and organ: training and test datasets, left lung, right lung, trachea, spleen and left kidney. Note that the DICE similarity coefficients are high or very high, with a relatively small standard deviation. Our method achieved an overall DICE score of 92.8 and was ranked first among the five methods that participated in the challenge. Fig. 4 shows four representative examples of the multi-organ segmentation results. 


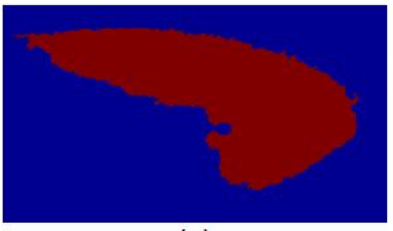

(a)

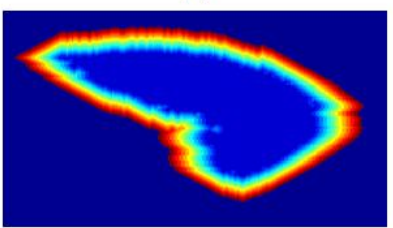

(c)

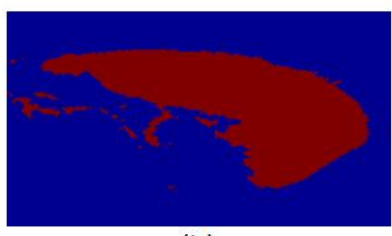

(b)

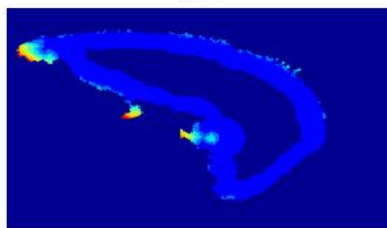

(d)

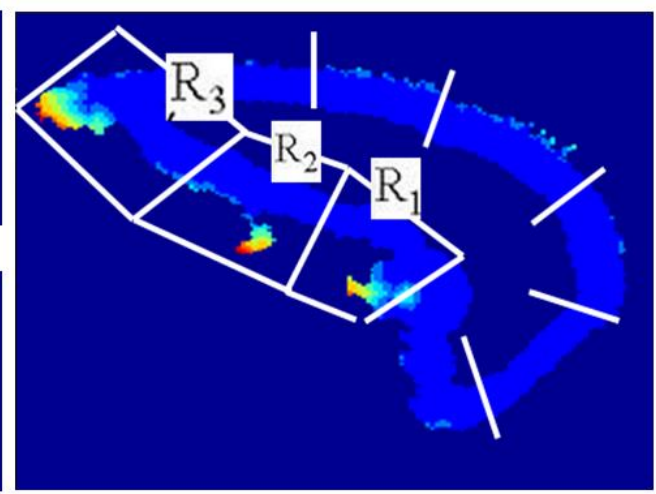

(e)

Figure 3: Illustration of step 4, 3D region growing, on the right lung: a) initial LACSS; b) adjacent LACSS; c) distance map; d) intersection image of the adjacent LACSS and distance map, and e) windows along the contour.

\begin{tabular}{|c|c|c|c|c|c|}
\hline Training dataset & Left Lung & Right Lung & Trachea & Spleen & Left Kidney \\
\cline { 2 - 6 } & 97.4 & 97.6 & 79.1 & 89.2 & 92.8 \\
\hline \hline \multirow{2}{*}{ Test dataset } & Left Lung & Right Lung & Trachea & Spleen & Left Kidney \\
\cline { 2 - 6 } & 97.9 & 97.0 & 85.6 & 93.4 & 90.2 \\
\hline
\end{tabular}

Table 1: Results: Mean Dice similarity coeffficient and standard deviation for the training and test datasets on each organ.
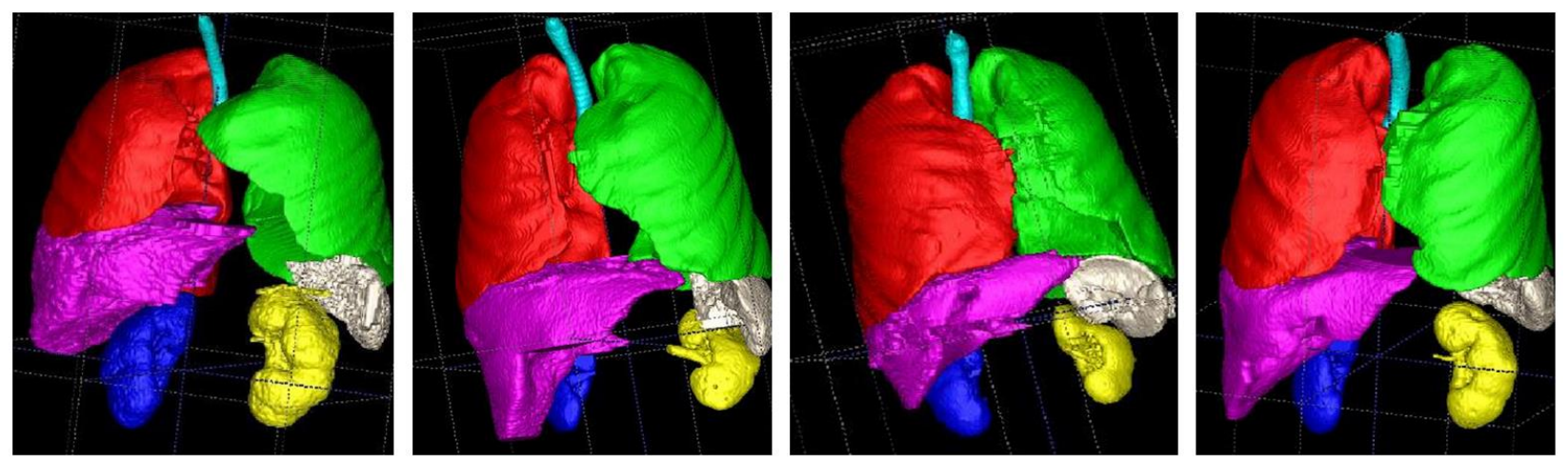

Figure 4: Multi-organ segmentation results of four representative datasets of the VISCERAL Anatomy2 Challenge.

\section{Conclusions}

We have developed a generic framework for the segmentation of ventral body cavity organs in CT scans. Our approach consists of four-step pipeline method that takes into account prior information about the locations of the organs and their appearance in CT scans. We have shown that the method is applicable to a variety of ventral body cavity organs including the trachea, the left and right lungs, the spleen, and the left kidney.

Current and future research is incorporating other structures, including the right kidney and the liver. We are also extending the $3 \mathrm{D}$ region growing step to include different smoothing criteria in different regions of the organ, to eliminate and avoid leakage to neighboring organs. 


\section{References}

[1] Langs G, Hanbury A, Menze B. VISCERAL: towards large data in medical imaging challenges and directions. Proc. MICCAI LNCS: Medical Content-Based Retrieval for Clinical Decision Support, pp 92-98, 2013.

[2] Rubin DL, Akgl CB, Napel S, Beaulieu CF, Greenspan H, Acar B. Content-based image retrieval in radiology: current status and future directions. Journal of Digital Imaging, pp 24-32, 2011.

[3] Schmidt G. et al. Cognition network technology for a fully automated 3D segmentation of the liver. Proc. MICCAI Workshop on 3D Segmentation in the Clinic: A Grand Challenge, pp 125-133, 2007.

[4] Mharib AM, Rahman A, Mashohor S, Binti R. Survey of liver CT image segmentation methods. Artificial Intelligence Review, pp 37-42, 2012.

[5] Sluimer I, Schilham A, Prokop M, van Ginneken B. Computer analysis of computed tomography scans of the lung: a survey. IEEE Transactions on Medical Imaging Vol 25(4), pp. 385, 2006.

[6] Freiman M, Kronman A, Esses SJ, Joskowicz L, Sosna J. Non-parametric iterative model constraint graph min-cut for automatic kidney segmentation. Proc. MICCAI, LNCS 3217, pp. 73-80, 2010. 